\title{
Enhancing the Performance of Current Online Education System- A Study of Cloud Computing and Virtualization
}

\author{
Basheer Riskhan, Raza Muhammed \\ School of Computer Science, Huazhong University of Science and Technology, Wuhan, China \\ Email: riskhan@yahoo.com
}

Received 30 September 2015; accepted 27 October 2015; published 30 October 2015

Copyright (C) 2015 by authors and Scientific Research Publishing Inc.

This work is licensed under the Creative Commons Attribution International License (CC BY). http://creativecommons.org/licenses/by/4.0/

(c) (7) Open Access

\begin{abstract}
Online Education (OE) system is an effective and efficient way to perform the education in all sectors of government and non-government educational organization. Low performance and minimum speed are major overhead in the current ongoing $\mathrm{OE}$ system due to the increase of users and some system issues. Base on the previous study and recent practical issues, a model is proposed to Enhancing the Performance of Online Education System (EPOES) to examine the bare metal virtualization, isolation and virtual machine templates. Bare metal virtualization has led the native execution, isolation isolated the running application and Virtual Machine Template has help to increase efficiency, avoiding the repetitive installation and operate the server in less time. The proposed model boosts the performance of the current $\mathrm{OE}$ system, and examines the benefits of the adaptation of cloud computing and virtualization which can be used to overcome the existing challenges and barriers of the current OE System.
\end{abstract}

\section{Keywords}

Bare Metal Virtualization, Cloud Computing, Isolation, Virtual Machine Template

\section{Introduction}

The demand for computer usage has increased significantly over the past few decades. As a result, Online Education (OE) conducted by web based technology has become a crucial part of education and it has many issues based on inefficient management, slow process, less performance and security vulnerabilities [1]. To overcome the above overhead, the proposed model introduced is called "Enhancing the Performance of Online Education

How to cite this paper: Riskhan, B. and Muhammed, R. (2015) Enhancing the Performance of Current Online Education System-A Study of Cloud Computing and Virtualization. Journal of Computer and Communications, 3, 43-51. 
System (EPOES)”. Bare metal virtualization, isolation and virtual machine templates are the cloud computing and virtualization technologies which are suggested to implement to that model. Further this model will allow the user feasibility for portable network device (PND).

In the past decades, the most flaws and overheads were improved; education providers have begun to show more and more interest in web based tools including Web CT and web 2.0 [2] for the use of OE system. But even with an upgrade software system, there still have some disabilities such as security vulnerabilities, virus attack, less bandwidth, fixed location, using only computers as the main source to access to OE, limited accessible space, limited funds, maintained by the administration, frequent software update, high cost of hardware and software. As a result IT administrators face performance and speed as a major concern as the system fails to provide optimal performance and speed with the increase in the number of OE users. Enhancing the performance of present $\mathrm{OE}$ system is our main objective of this paper and issues regarding performance can be overcome by proposing the model EPOES with using new technologies such as bare metal virtualization, isolation and virtual machine templates based on cloud computing.

Cloud computing technology is the most preferred method as it is one of the fastest growing technologies in IT. It is Internet (cloud) base development and use of computer technology (computing), whereby dynamically scalable and often virtualized resources are provided as a service over the Internet. Hiding and abstraction of complexity, virtualized hardware and efficient distribution of resources are some of the key benefits of cloud computing [3]. Cloud computing also reduces the cost of IT, provides resource as per demands, reduces the license constrain, increases the agility, maximizes the speed, shares and allocates hardware devices. Google, Amazon, Yahoo and Microsoft are some of the cloud computing service providers in the current market; they support the OE system by providing sufficient amount of memory and applications sharing [4]; they provide the services to access and develop the OE applications in the easy manner. Although cloud computing is still at the early stage of its development in the education sector, there are many more challenging issues waiting to be explored using this versatile system.

Implementation of new technologies based on virtualization and cloud computing will allow the user to access OE system anywhere with the aid of PND such as smart phones, PDAs, surface and iPad touch. It also has a positive impact on the high performance of OE. In the case of insufficient memory, CPU, application or storage, cloud has the technology to fulfil the overheads according to the demand. Whereas, virtualization has a lot of potential for virtualized software to reduce cost, increase fast access, unify management, improve IT responsiveness, increase IT utilization and deliver IT seamlessly and securely. The above benefits given the support upgrade the drawback of OE system.

\section{Related Work}

Newly proposed technology mainly focuses on virtualization and cloud computing technology. It has derived from grid computing, distributed computing, utility computing, parallel computing and other computer technologies [4]. Cloud computing shares memories and resources, as compare grid computing wants to solve the task of memories and resources. Like cloud computing and virtualization technology mostly relate with virtualization compare with Grid computing. In order to minimize the human factor in the examination process of OE, the application database technology will be a question gathered into the database before each test composed of questions from the exam papers extracted, greatly reducing the workload of teachers to effectively reflect a fair test sex [5]. This is the method using for conducting examination in manually and existing online architecture. The new model is introduced to import to the cloud computing technology with adding some new features such as resource sharing, centralized hardware and software, reduce the cost, access software's with flexibility, access anywhere, anytime and anyplace, without caring updates and security. But Security, sufficient Internet access, bandwidth and acceptance of decision makers in the institutions are some of the main factors that are considered as implementation of cloud computing in the academic institutions [6].

Most of cloud computing platforms are based on virtualized environments [3]. Virtual machine user is able to run a variety of operating systems and environments as needed by the applications. Virtualization allows user to isolate workloads, improving security and reliability [7]. According to the above statement isolation and improvement of the security from hackers are given the improvements in performance of the OE system. Due to that, the proposal to implement the isolation technology to the EPOES model by support of the virtualization. From the research, the new model EPOES is introduced as a solution to the previous overheads of the current OE system. Further this model enables to prevent the unauthorized access, improve the personal identity tech- 
niques and challenge the existing architecture of OE.

\section{Analysis of the Current Performance of Online Education}

OE improves the efficiency and accuracy of the overall education process. In recent years most of the higher institutions are showing interest to migrate from traditional pen and paper based education pattern to OE system. However, the present OE system architecture has lots of drawbacks such as performance, processing speed, security and dishonesty of students, such as coping answers of others, searching the internet for answer, storing the data's on the local computer and using for the future exam purposes, changing the answer, discussion of answers through the email and mobile phone [8].

Figure 1 shows the ongoing architecture system which follows most of the OE system such as Learning Management System, Moodle. This architecture processed fully based on web technology with some disabilities. The main drawback of this current model was low performance. By implementing new technologies suggested in this paper can improve the advance performance of OE system in the future.

\section{Challenges of Current OE System}

As technology has improved, the need for OE has also increased in recent years due to various reasons. The drawbacks in the current $\mathrm{OE}$ have caused slight hesitation among providers to choose it as an educational tool. The following will discuss the drawbacks of the OE system.

\subsection{Band Width and Internet Speed}

Bandwidth and Internet Speed are the backbone for any good communication between client and server which are both technically different. Bandwidth refers to the maximum amount of data that can be supported or transferred through the channel within a fixed amount of time through the network [9]. Therefore, less Bandwidth would cause the overhead to move the data from two geographically separated networks. Speed performance relies on a large bandwidth pipe and routing implemented in a network. In the present OE system, the clients connect with the LAN Server and request to move through that Server. Bandwidth also performs an important role when the data is moved from the LAN network. The problem arises when performing bandwidth and data intensive tasks such as moving large data sets, performing replications for high availability and backup purposes, or data mining or ETL activities [10]. If the availability of bandwidth is high, then the compatibility of data movement becomes faster. Generally all the computer labs operate in a smaller bandwidth range, which results in a less performance of OE. Internet speed is the time taken for the data to reach the destination from the source. The data transfer speed varies depending on the implemented technology. Therefore, the Bandwidth and Internet speed dominate the performance of the OE Process.
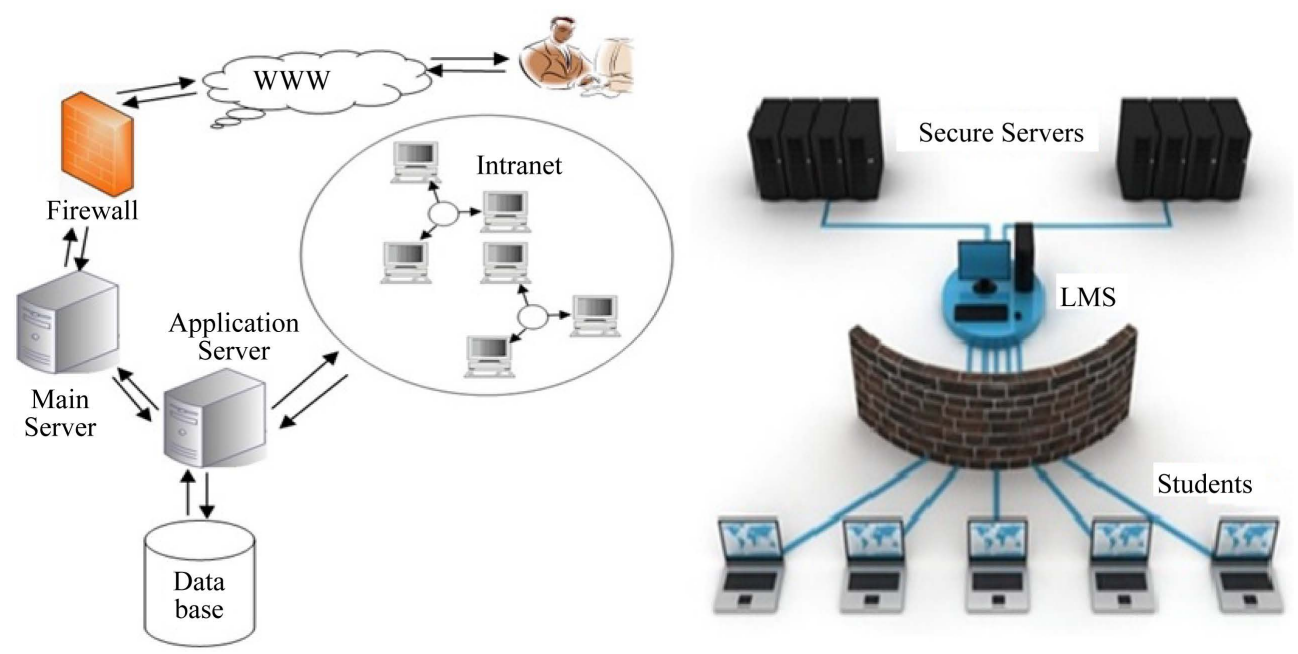

Figure 1. Technical architecture of current OE system. 


\subsection{Server Workload and Efficiency}

Workload is the amount of processing that the computer has been set to perform at a given time. The workload consists of a number of application programs running in the computer, with a number of users connected to and interacting with the computer's applications [11]. As the number of online education user increase, the amount of processing and the amount of application programming running at the present server also increases, causing an increase in the server workload. The protocol used to send the data, network conjunction and server efficiency are factors, dependent on the network speed [9]. For example, in the scenario of attempting to transfer data from one location to another, the data transfer is considered advanced when done through a faster server than to a slower server. Given an optimal internet speed, a slow server would slow the data transfer in comparison to the fast server where the rate of transfer is high. Therefore, the performance of the OE system is dependent upon the server workload and server efficiency.

\subsection{One Terminal Access}

One terminal access describes the flow of signals to one single port. When the LAN users login to start the OE process, all of the user requests are directed straight to one LAN server. This process leads to a queue in the server while the requests made by the users begin to process through using FIFO or LIFO algorithm. Same scenario is applied throughout the other servers involving OE operation. Process time of these servers depend on the amount of workload submitted by the number of users through individual terminals. Once all of the tasks are received from LAN server, the data gets moved to a single terminal. This again creates another set of queue causing the process time of OE slower and the performance going to less.

\subsection{More Servers inside the OE Architecture}

Figure 1 clearly explore, that the Current OE systems were implemented through different servers like web server, database server, admin server, ISP Server and LAN server to uplift the standard of OE service between users and destination. Because all of the servers are depended on each other, failure of one server will cause the failure of OE system. Furthermore, the servers engaged in doing their own separate tasks require a larger bandwidth range to let the data travel through all other servers, as a result it performance down the OE process.

\subsection{Traditional Security System and IP Security}

Each server has its own security system for receiving and sending the request. Current OE system uses the traditional security systems such as data encryption, firewalls and data signature. High security and power hungry software's can slow down the performance of education system. Network Sniffers, IP Spoofing, Connection Hijacking, Data Spoofing are the main problems in the IP Security and an attack on a network is created in order to gain unauthorized access.

\subsection{Fixed Device Access and Physical Disaster}

Fix device access is another drawback of this system. At present remote access is a rising concept in IT. All the devices can be connected with the help of remote access, providing great convenience to the user. Introduction of portable network devices such as smart phones, PDAs, Surface and iPad in the current market has piqued the interest and usage of public. Although these various devices those have been introduce into the market, the operation of $\mathrm{OE}$ is still conducted only via the use of computers. This is also hidden technique to slow performance process of an OE. System failure of OE can also be caused by natural disasters such as fire and flood. Damages caused by these events are equally significant as the damages caused by software failure and human Errors due to above reasons the speed of process of online education might be going slow. If any early sign regarding above failures it's helpful to minimize the impact of a possible failures [12].

\subsection{Malware Attack}

Malware is software that infects and damages a computer system without the owner's knowledge or permission. Malware includes computer viruses, computer worms, Trojan horses, spyware, and crime ware [13]. A virus infection or security breach on the server can lead to corrupting of data, shutting down of documents, Restart and 
Overload the server. Servers have run out the memory and stop the operating services in the servers, reduction in server efficiency and server breakdown. Thus, large amount of funding is required to maintain and run the servers securely from the virus attack. Furthermore virus intent to processing more unwanted task inside the server, reduce the server efficiency. One of the major concerns in the OE operation is the malicious programming attacks on the networks by hackers. Once the worms are left in a server, they can replicate and spread themselves in a short period of time. This causes the user to be automatically affected by the entire wired or wireless device excluding the proper production device which is connected to the LAN or network. Slow internet, slow network connection and slow data transfer are signs of worm infection [13]. Due to that above causes, the OE system shows less performance.

\section{Methodology of the Model of Enhancing the Performance of Online Education System (EPOES)}

In this section, the EPOES model is introduced to minimize the above obstacles. This model contains physical server, physical resources and virtual machine which will be briefly discussed in sub sections, cloud computing and virtualization technologies were implemented to that model. The users access to the EPOES model remotely by using any PND.

\subsection{Implement Resources}

In this section recommendations to implement some resources to the EPOES from the previous study and observation like Physical Server, Physical Resources and Virtual Machine based on cloud computing and virtualization, as they both have an inter-relationship. Virtualization is the key technique for Cloud Computing. Virtualization is a software that manipulates the hardware and cloud computing is the service that results from the manipulation. Virtualization is a foundational element that helps cloud computing deliver on its value. Cloud computing is currently on demand as it provides share of computing resources, software and data as a service [14]. Through virtualization and other resource sharing mechanisms, cloud computing can dramatically reduce the examination cost, increase fast access and create virtual machines. When the request is received, the physical server has the capability to create and execute the Virtual machine automatically and import the physical resources according to the user demand, based on Cloud computing.

\subsubsection{Physical Server (The Host)}

This physical server forms the public cloud environment, provides the virtual machine and controls its physical resources [15]. The public cloud environment provides the resources as Software and hardware according to the demand of the user, and executes the OE application interface.

\subsubsection{Physical Resources (Hardware)}

Figure 2 shows, the physical resources consist of CPU, memory, network, OS and etc. These resources will be allocated to the virtual machine.

\subsubsection{Virtual Machine}

The virtual machine is the virtual computing platform on a Physical server, by virtualized software and it is directly connected to the physical hardware shown on Figure 2.

\subsection{Implement New Techniques}

Consolidation, Isolation and Migration are the main factors in the system level virtualization and it will be used for the number of reasons [16]. The main objective of this paper is to demonstrate, how the process of performance of the OE System can be improved by the use of cloud base virtualization system. So Bare metal virtualization, Isolation and Virtual machine templates are the techniques of cloud base system level virtualization and they are the most appropriate techniques to increase the performance of present OE system.

\subsubsection{Isolation}

Isolation is the property of virtualization. It is an approach to improve the performance of the process and 


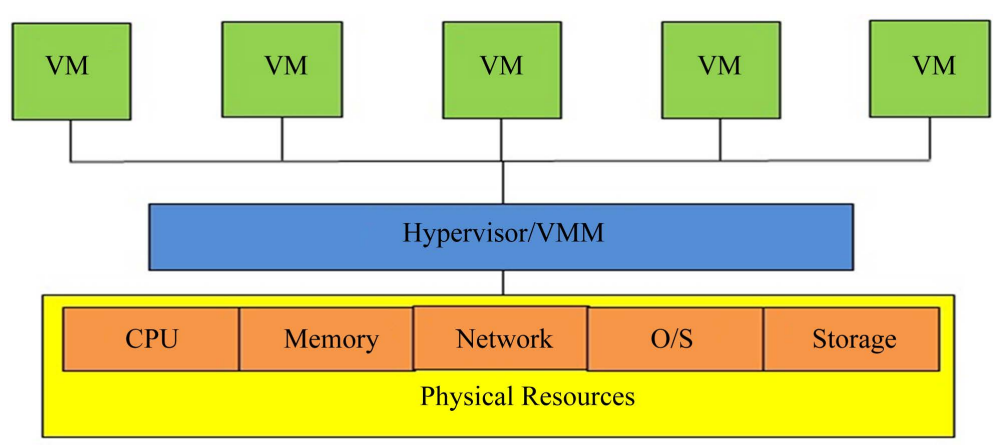

Figure 2. Virtual computing platform environment.

reliability of the system by isolating the execution environment for resources, application and OE related database [16] shown on Figure 3. This isolation enables multiple virtual environments to run securely while sharing the resources and applications [17]. Generally the virtual machines are the container that consists of resources, application and $\mathrm{OE}$ related database and they are designed to be isolated from one another.

If any execution failure occurs in any virtual machine, the other virtual machine on the same host will continue to run due to those stated reasons of isolation. However, resources or application failure has no effect on the performance of the virtual machine, the ability to access the other virtual machine or the operation to access the required resources [18]. Each virtual machine is isolated through the virtualization technology while running on the same hardware. Virtual machines share physical resources such as CPU, memory, I/O device and application, in order to utilize virtual machine isolation to concurrently execute several virtual machines in a single server. To protect the virtual machine from attracting virus or worm, safeguards such as antivirus software, firewalls can be applied to the physical machine [18]. Virtual machines requiring hypervisor/VMM and batch system can effectively map this view of the system [7].

\subsubsection{Bare Metal Virtualization}

Hypervisor is a program that is included in the host operation system. It can be activated through several virtual machines using a single hardware. Hypervisor is also known as a Virtual Machine monitor. This controls the host resources and allocates the required resources to each virtual machine. Figure 4, explores the categories of hypervisors in two types as bare metal virtualization and hosted virtualization [16]. In bare metal virtualization, VMM and VM are direct mapping with the hardware resources and the process is comparable to the native execution. Hypervisor is used only for bypass [19] as the guest OS running within the virtual machine, manages and maps the resources in order to enable the execution of an application [7].

Hosted virtualization Hypervisor only appears between a host OS and VM. The Hypervisor mapping shares resources and application according to the requirement of VM. In a hosted virtualization between host OS and VM, VMM is the bottle-neck of this proposed platform. This approach is the most suitable for the development purpose [18]. Direct connection with Physical Resources help to sustain the time conception, processing speed and high performance that are the key factors of OE system. Thus, the recommended technology for the OE is system is bare metal virtualization.

\subsubsection{Virtual Machine Templates}

Virtual machine template functionality is developed in VM ware Infrastructure 3. The key benefits of this is that it avoids repetitive installation, uses less processing time and is efficiency [20].

Assume 2 students are to sit for an exam. In the Manual Installation of Virtual Machines, it creates two virtual machines as in Figure 5 and these process takes minimum of 5 steps for a single user. For 2 users this will be minimum of 10 steps, as each task is repeated by every user, it is time consuming. Whereas, Template Based Deployment of Virtual Machines creates only one virtual machine and it can reduce 10 steps to 7 steps for the entire process as in Figure 5. When the users increase, large differences occur within the process steps between Manual Installation of Virtual Machines and Template Based Deployment of Virtual Machines. Therefore, less time and faster performance occur through avoiding the repeated installation. 


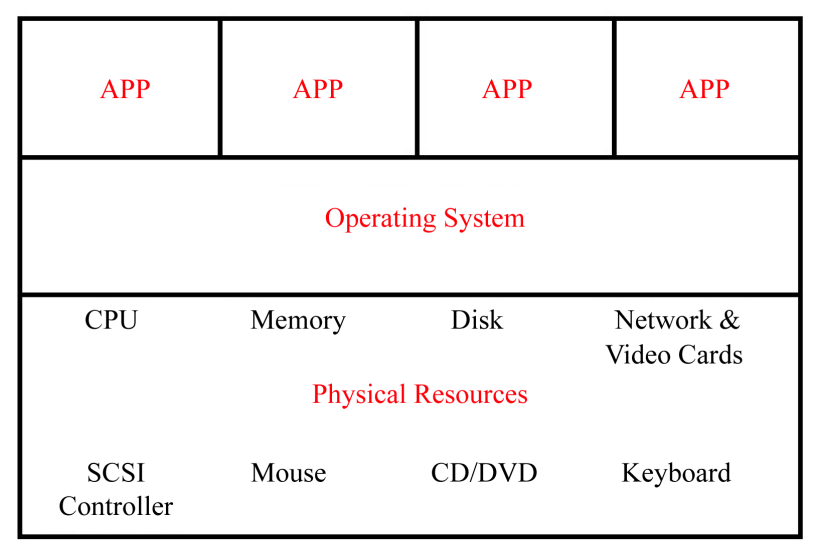

Figure 3. Virtual machine isolation.

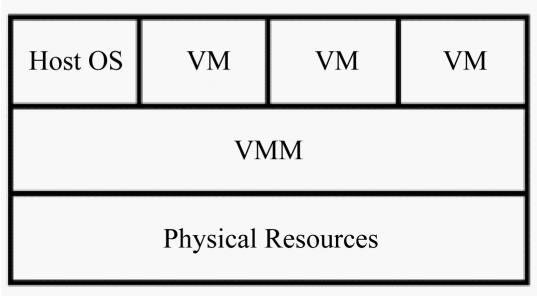

Bare Metal Virtualization

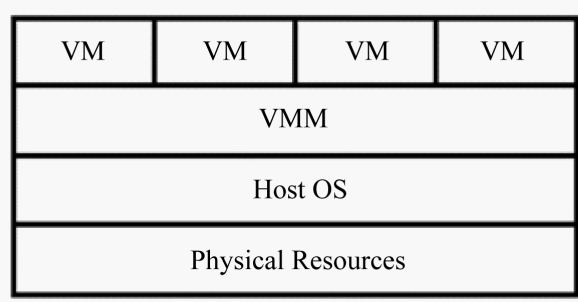

Hosted Virtualization

Figure 4. Types of virtualization.

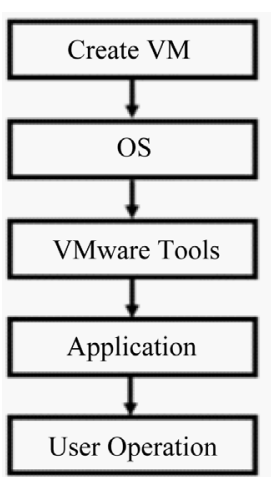

Manual Installation of VM

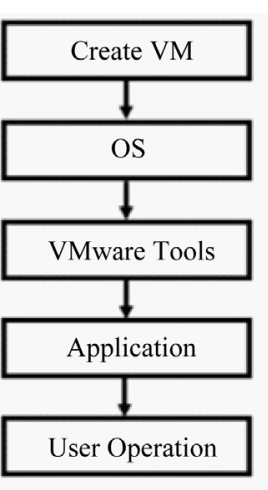

Figure 5. Manual and templates base VM.

\section{Impact of Cloud Computing and Virtualization for the Current Performance of Online Education}

Main key challenges with the current OE system are bandwidth, server workload, one terminal access, malware attack, more servers, IP security and fixed devices. Bare metal virtualization, Isolation and Virtual Machine Template are the technologies that have been proposed to implement the current system in order to avoid slow performance. The following section will discuss how the proposed technologies can be used to resolve the stated challenges.

For a data to be successfully transferred from one location to another (client to ISP, ISP to ISP and ISP to Server), more bandwidth and good network speed are crucial. Cloud providers usually have high bandwidth and high network speed due to good technologies being used for communication media and for other networks [21]. Recent "Computerworld Report" said that the cloud support to the lack bandwidth. Server workload is reduced through isolation, where the share resources and application in a single VM for a single user is separated. Con- 
sider most students accessing the OE system at the same time by directly connecting with the server. In this scenario, a lot of bottle-neck is created to both the server and student. This leads to slow down the performance, increase the chances of hacking questions, time limitation and there is a risk of striking the server. In order to overcome the above drawbacks, the present OE System uses the Isolation technique based on cloud based virtual environment which is one of the good solution.

One terminal access and fixed device issues can be solved by using cloud base virtualization technology. In the current technology world the portable network devices can provide convenient ways to access the network at anywhere and at anytime. Technology for these portable network devices has been extended continuously by $2 \mathrm{G}$, 3G, 4G and etc. So by letting portable network devices access OE system, the issues with the fixed devices can be minimized. Also by using the portable devices, the users avoid the need to be at a fixed location such as computer labs and etc, help to reduce the one terminal access. Consider 10,000 users accessing the OE at the same time. During this occasion, the server's workload will increase along with power consumption, building space and amount of available resources will increase. Thus by using virtualization technique, it enables the users to access the $\mathrm{OE}$ from any location and at any time, while keeping the number of computers, building space and maintenance cost down. If any disabilities were to occur in the portable network devices due to power consumption, memory and hardware resources then the cloud computing will provide the necessary assistance with the needed on demand.

Bare metal virtualization is one of the techniques used by virtualization that has a direct connection between the Virtual Machine and Physical resource. This will assist in getting the native execution in the virtual machine resources. Therefore the number of users is not a concern, according to the number of users and the server machines create the virtual machines and share the device with the native execution. In addition, cloud computing solves the IP Security issue as the end user has no knowledge of the underlying technology [22]. The end user is one who uses the product after it has been fully developed and marketed which includes Hackers. As no knowledge of the designation point (IP) in the cloud computing in available, it causes the hackers to face major difficulties in creating interruption to the IP Security.

Virtual machine templates provide the opportunity to get high benefits with less VMs via the use of image templates. Time conception is another key concept in the OE system, as in a usual system 5 VM is required for 5 OE users to access the OE applications. However, by using Virtual machine templates technique, 5 users can access the $\mathrm{OE}$ in a single VM through image templates. The process is very fast, repetitions are avoided, reduced issues of Malware Attack and fewer servers are use among the benefits. If the above technique is implemented to the proposed model EPOES using cloud based system level virtualization, the barriers mentioned can be overcome.

\section{Conclusion}

A number of difficulties and constrains such as low performance and less speed have been raised in the current OE system due to some barriers and increased the number of users. The paper focused on low performance of OE system and this issue could be resolved by the proposed model EPOES with the implementing some new technology with the support of system level virtualization and cloud computing technology. Bare metal virtualization, isolation and virtual machine templates are the important techniques required to implement the proposed model. It provides the opportunity to enhancing the performance of OE system by sharing physical resources with the native execution, reducing the IP security issues, mitigating virus attacks and letting portable network devices to access the OE system in anytime, phase.

\section{References}

[1] Sarrayrih, M.A. and Ilyas, M. (2013) Challenges of Online Exam, Performances and Problems for Online University Exam. IJCSI International Journal of Computer Science Issues, 10.

[2] Rogers, C.F. (2006) Faculty Perceptions about e-Cheating during Online Testing. Journal of Computing Sciences in Colleges, 22, 206-212.

[3] Tian, W.H., Sun, X.S., Jiang, Y.Q. and Wang, H.Y. (2013) CRESS: A Platform of Infracture Resource Sharing for Educational Cloud Computing. Communication, China, 10, 43-52. http://dx.doi.org/10.1109/CC.2013.6623502

[4] Leloglu, E., Ayav, T. and Aslan, B.G. (2013) A Review of Cloud Deployment Models for E-Learning Systems. Dependable Systems and Networks (DSN). 3rd Annual IEEE/IFIP International Conference. 
[5] Hang, B. (2011) The Design and Implementation of On-Line Examination System. ISCCS 11 Proceedings of the 2011 International Symposium on Computer Science and Society, Kota Kinabalu, 16-17 July 2011, 227-230. http://dx.doi.org/10.1109/ISCCS.2011.68

[6] Mokhtar, S.A., Ali, S.H.S., Al-Sharafi, A. and Aborujilah, A. (2013) Cloud Computing in Academic Institutions. ICUIMC 13 Proceedings of the 7th International Conference on Ubiquitous Information Management and Communication, 2. http://dx.doi.org/10.1145/2448556.2448558

[7] Vallee, G., Naughton, T. and Ong, H. (2008) System-Level Virtualization for High Performance Computing. 16th Euromicro Conference on Parallel, Distributed and Network-Based Processing, Toulouse, 13-15 February 2008, 636-643. http://dx.doi.org/10.1109/PDP.2008.85

[8] Naresh, C., Deepthi, C.H. and Shekhar, T.P. (2011) A Novel Approach to Enhance Security for Online Exams. International Journal of Computer Science and Technology, 2, 85-90.

[9] Ferro, G. (2015) Home. The Difference between Bandwidth and Speed. http://etherealmind.com/basics-difference-bandwidth-speed/

[10] Treadway, J. (2010) Home. Optimizing the Cloud: Solving the Bandwidth Issue. http://www.cloudtp.com/2010/08/19/optimizing-the-cloud-solving-the-bandwidth-issue/

[11] Center, Search Data (2006) Workload Definition. [Online] http://searchdatacenter.techtarget.com/definition/workload

[12] Microsoft (2015) Library, Causes of Failure. Microsoft System Center. [Online] http://technet.microsoft.com/en-us/library/cc180043.aspx

[13] Logan, D. (2013) Tech. What Is Malware? [Online] http://www.ehow.com/facts_4857164_what-is-malware.html

[14] Angeles, S. (2014) Technology. Virtualization vs. Cloud Computing: What's the Difference? [Online] http://www.ebates.com/index.htm?src=marketing\&acct=tracsion\&camp=paid\&utm source=tracsion\&utm medium=b anner\&utm_campaign=paid\&site $=104$

[15] Navarro, T. (2013) Home. What's the Difference between Virtualization and Cloud Computing. [Online] https://www.computenext.com/blog/the-difference-between-cloud-computing-and-virtualization/

[16] Scott, S.L., Vallee, G., Naughton, T., Tikotekar, A., Engelmann, C. and Ong, H. (2008) System-Level Virtualization Research at Oak Ridge National Laboratory. Oak Ridge National Laboratory, Oak Ridge.

[17] Sun, D.W., Chang, G.R., Guo, Q., Wang, C. and Wang, X.W. (2010) A Dependability Model to Enhance Security of Cloud Environment Using System-Level Virtualization Techniques. Pervasive Computing Signal Processing and Applications (PCSPA), 305-310.

[18] VMware (2015) VMware vSphere Introduction. VMware vSphere 4-ESX and vCenter Server. [Online] https://pubs.vmware.com/vsphere-4-esx-vcenter/index.jsp

[19] Liu, J.X., Huang, W., Abali, B. and Panda, D.K. (2006) High Performance VMM-Bypass I/O in Virtual Machines. Computer Based Learning Unit, University of Leeds, 1-7.

[20] Dodge, J. (2006) Introduction. Virtual Center 2. [Online] http://www.vmware.com/pdf/vc_2_templates_usage_best_practices_wp.pdf

[21] Momjian, B. (2015) Home. Increasing Website Bandwidth Using Cloud Service. [Online] https://momjian.us/main/writings/pgsql/cloud bandwidth.pdf

[22] Hurwitz, J., Bloor, R., Kaufman, M. and Halper, F. (2012) Cloud Computing for Dummies. John Wiley \& Sons, Inc., New York. 